Ludwig A. Th. Greif, Stefan T. Jagsch, Markus R.Wagner, Andrei Schliwa

\title{
Tuning the Emission Directionality of Stacked Quantum Dots
}

Journal article | Accepted manuscript (Postprint)

This version is available at https://doi.org/10.14279/depositonce-10474

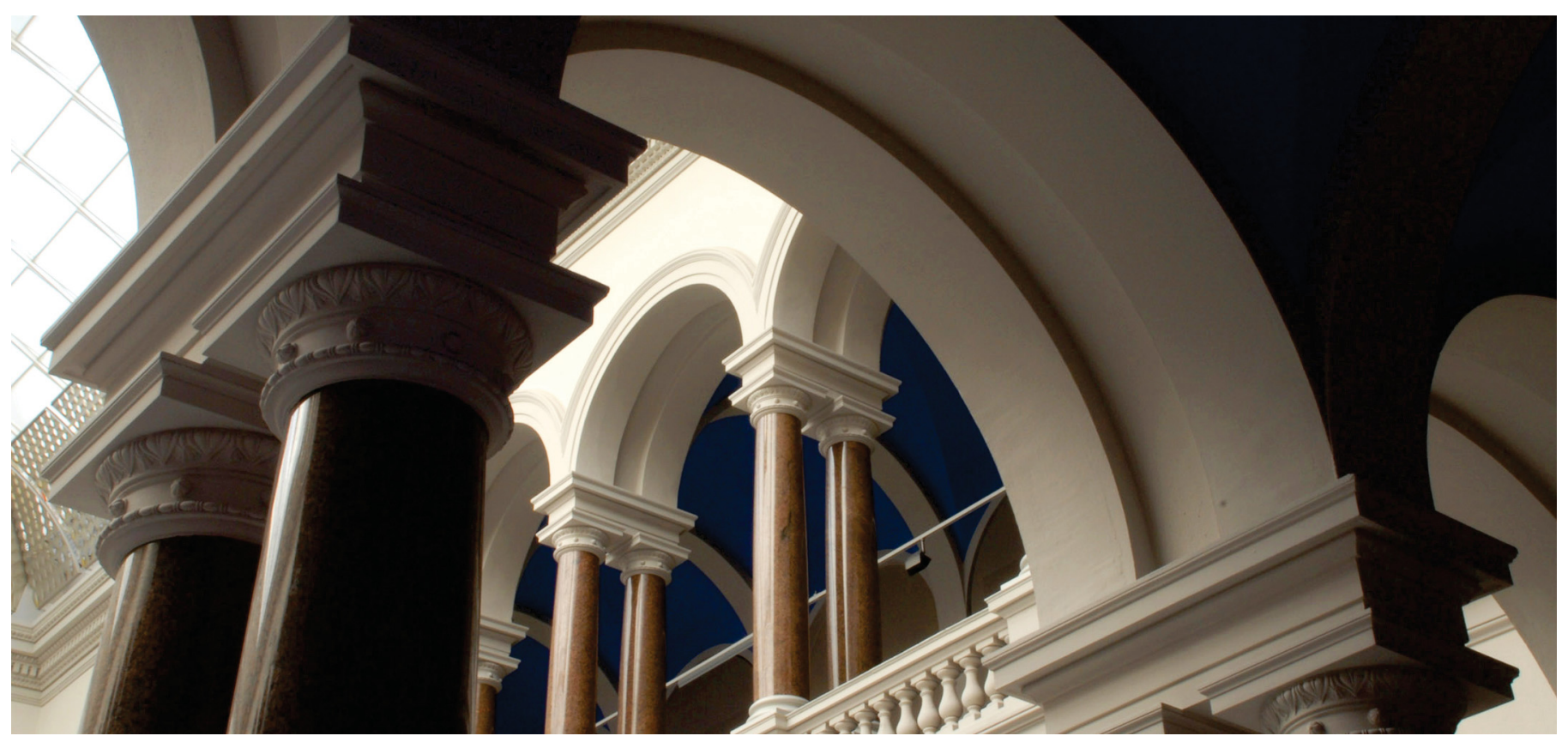

Greif, L. A. Th., Jagsch, S. T., Wagner, M. R., \& Schliwa, A. (2018). Tuning the Emission Directionality of Stacked Quantum Dots. ACS Photonics, 5(12), 4838-4845. https://doi.org/10.1021/acsphotonics.8b00956 


\title{
Tuning the Emission Directionality of Stacked Quantum Dots
}

\author{
Ludwig A. Th. Greif,* Stefan T. Jagsch, Markus R. Wagner, and Andrei Schliwa \\ Technische Universität Berlin, Institut für Festkörperphysik, Hardenbergstraße 36, 10623 \\ Berlin, Germany \\ E-mail: lath@physik.tu-berlin.de
}

\section{Abstract}

The emission directionality of stacks of coupled quantum dots (QDs) is investigated within the framework of 8-band $\boldsymbol{k} \cdot \boldsymbol{p}$-theory including strain and strain-induced piezoelectricity up to second order. Using an artificial cuboidal QD we show that the degree of radiation anisotropy can be tuned from $-33 \%$ to nearly $+60 \%$ via the structure's vertical aspect ratio. We then demonstrate that these findings can be transferred to stacked InGaAs quantum dots whose emission directionality is tailored (i) via the interdot coupling strength given by the separating barrier width, as well as (ii) the number of stacked QDs. Our results enable the design and optimization of top and edge emitters based on stacked QDs.

\section{Keywords}

self-assembled quantum dots, radiation pattern, $\boldsymbol{k} \cdot \boldsymbol{p}$-theory, light-hole groundstate, valenceband mixing

Quantum dots (QDs) usually exhibit a pronounced spatial anisotropy of emission. ${ }^{1-3}$ Controlling the emission directionality constitutes a promising way of optimizing photonic devices. Possible applications include energy efficient lighting and display technology where deterministic alignment of the emission direction with respect to the cavity can increase the gain, ${ }^{4}$ while simultaneously decreasing losses into leaky modes. Such alignment would also improve the properties of QD based microlasers reducing the threshold current, ${ }^{5}$ increasing the modulation bandwidth ${ }^{6}$ and reducing the mean first passage time. ${ }^{7}$ Due to their "atom-like" emission properties, QDs also play an important role in quantum optics and the emerging field of quantum information technology, where the extraction efficiency is a key parameter that can benefit from control over the emission direction. ${ }^{8,9}$

Considerable effort is put into investigating and controlling optical properties of QDs. The enhanced density of states and the reduced electron scattering, ${ }^{5}$ as well as band gap engineering via material, strain and size control ${ }^{10}$ all together lead to an adjustable gain material with high temperature stability and narrow linewidths. ${ }^{5,11-13}$ In several works, the influence of coupling between QDs and Yagi-Uda nanoantennas, ${ }^{28}$ quantum-/nano-wires ${ }^{29,30}$ and photonic crystals ${ }^{31,32}$ with respect to the direction of predominant emission is analyzed. The central question in these works is "how can the emission directionality of QDs be designed through interaction with their environment? ".

In contrast, we focus on how the emission direction can be controlled through the structural properties of the emitter itself, using the intrinsic parameter of the emitter's vertical aspect ratio $(A R)$. To this end, material and growth dependent limitations to the shape of single QDs can be overcome by forming vertical stacks (or columns) of QDs, which can be achieved through strain induced self-alignment tech- 
niques. ${ }^{33}$ Thereby, ensembles with more than 30 vertically stacked QDs can be formed, ${ }^{14,34}$ where the interdot coupling strength is adjusted via the separating barrier width. If the interdot coupling is sufficiently strong, the ensemble constitutes a single confinement region with one common groundstate.

A large number of experimental ${ }^{1,14-20}$ and theoretical studies ${ }^{21-27}$ investigated polarization properties of stacked QDs in the InGaAs material system and the connection to strain distributions, valence-band splitting and state localization. Recently, Yuan et $\mathrm{al}^{35}$ considered the related question of how the quantization-axis of initially strain-free GaAs/AlGaAs-QDs can be adjusted by using external stressors. They found that the transition dipole moment, the valence-band mixing and oscillator strength of such QDs can be controlled via uniaxial stress engineering.

However, although the angular resolved emission intensity can be derived from the polarization resolved transition matrix moments presented in Ref. [21-27,35], a comprehensive presentation of the emission directionality of stacked QDs, acting as guideline for device design, is still missing.

We are not aiming to discuss the question of emission directionality in relation to the wide variety of parameters which exist for stacked QDs but want to reduce it to its most basic dependence - the $A R$ of the confinement region and the resulting biaxial strain in the region of wavefunction localization.

The paper is organized as follows: After outlining the details of our simulations we show that the $A R$ of a confinement region constitutes a useful parameter to tune its emission direction along the growth direction or the basis plane. We study three different series of QDs (Fig. 1). Series A consists of artificial cuboidal InAs-QDs which facilitates modeling arbitrary ARs. Subsequently, two series (B and C) of stacked InGaAs-QDs are simulated to show that the findings of series A can be transferred to realistic structures. The QDs of series B and C demonstrate that top emission as well as edge emission can be achieved by (i) adjusting their mutual coupling strength through the separating bar- rier width $w_{\mathrm{b}}$ (Series B) and (ii) the number of stacked QDs (Series C) as structural parameters.

Experimental data are scarcely available as it is difficult to prepare a stack of QDs such that emission can be detected in various propagation directions without disturbing effects like diffraction and reflection at the surface of the matrix material.

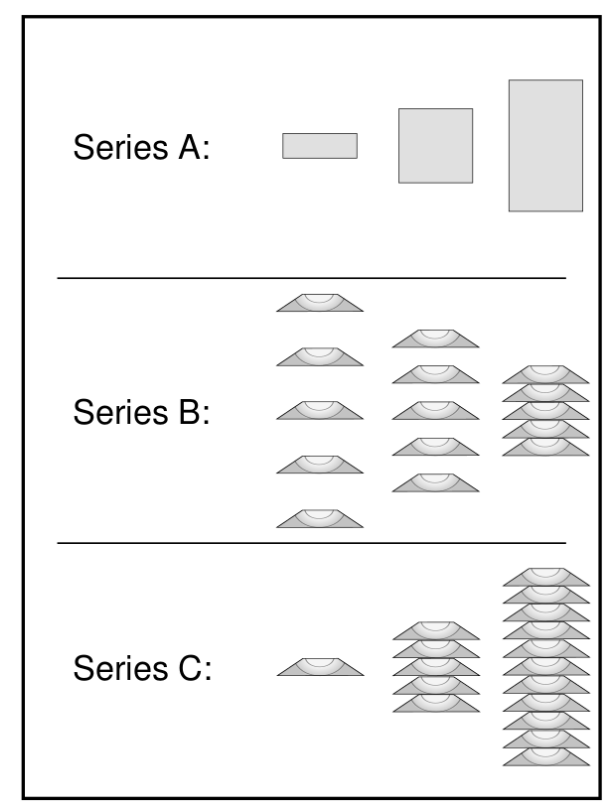

Figure 1: Scheme of the calculated structure series. Series A: nine InAs-QDs with $A R$ between 0.4 and 1.6. Series B: eleven stacks of five identical InGaAs-QDs with different barrier widths from 10 to 0 ML. Series C: 1 to 11 stacked identical InGaAs-QDs with a barrier width of $0 \mathrm{ML}$.

\section{Method of Calculation}

Simulations of stacks with up to 11 QDs including long range interactions like strain and piezoelectricity means to address multi-million atom problems. Analyzing series of such structures leads to a vast computational cost if atomistic techniques are applied. To reduce the computational cost significantly, all calculations were performed using an 8-band $\boldsymbol{k} \cdot \boldsymbol{p}$-model including strain and strain induced piezoelectricity. ${ }^{10,36}$ Strain calculations follow previous works ${ }^{37}$ using boundary conditions chosen in agreement 
with experimental findings such that no displacements in the bottom (001)-plane and along the directions orthogonal to the respective side facets is allowed. No restrictions are applied to the top (001)-plane. The numerical problem is solved using a product basis:

$$
|\Psi\rangle=\sum_{\mathrm{i}=1}^{8}\left|\phi_{i}\right\rangle\left|u_{\text {atom }, \mathrm{i}}\right\rangle
$$

with the envelope $\left|\phi_{i}\right\rangle$ and the eight atomic-like Bloch functions $\left|\boldsymbol{u}_{\text {atom }}\right\rangle$ :

$$
\begin{array}{r}
\left|\boldsymbol{u}_{\text {atom }}\right\rangle=[|s \uparrow\rangle,|x \uparrow\rangle,|y \uparrow\rangle,|z \uparrow\rangle, \\
|s \downarrow\rangle,|x \downarrow\rangle,|y \downarrow\rangle,|z \downarrow\rangle]^{t} .
\end{array}
$$

Considering the quantization with respect to the $z$-axis ${ }^{38}$ the set $\left|\boldsymbol{u}_{\text {atom }}\right\rangle$ can be transformed into a set of eigenstates of the spin dependent Hamiltonian: ${ }^{39}$

$$
\left[\begin{array}{c}
\left|c b_{1}\right\rangle \\
\left|h h_{1}\right\rangle \\
\left|l h_{1}\right\rangle \\
\left|s o_{1}\right\rangle \\
\left|c b_{2}\right\rangle \\
\left|h h_{2}\right\rangle \\
\left|l h_{2}\right\rangle \\
\left|s o_{2}\right\rangle
\end{array}\right]=\left[\begin{array}{c}
i|s \downarrow\rangle \\
\frac{|x\rangle+i|y\rangle}{\sqrt{2}}|\uparrow\rangle \\
\frac{|x\rangle-i|y\rangle}{\sqrt{6}}|\uparrow\rangle+\frac{2|z\rangle}{\sqrt{6}}|\downarrow\rangle \\
\frac{|x\rangle-i|y\rangle}{\sqrt{3}}|\uparrow\rangle-\frac{|z\rangle}{\sqrt{3}}|\downarrow\rangle \\
i|s \uparrow\rangle \\
\frac{|x\rangle-i|y\rangle}{\sqrt{2}}|\downarrow\rangle \\
\frac{|x\rangle+i|y\rangle}{\sqrt{6}}|\downarrow\rangle-\frac{2|z\rangle}{\sqrt{6}}|\uparrow\rangle \\
\frac{|x\rangle+i|y\rangle}{\sqrt{3}}|\downarrow\rangle+\frac{|z\rangle}{\sqrt{3}}|\uparrow\rangle
\end{array}\right]
$$

with the conduction-band (CB) functions $\left(\left|c b_{i}\right\rangle\right)$ and valence-band (VB) functions: heavy-hole $\left(\left|h h_{i}\right\rangle\right)$, light-hole $\left(\left|l h_{i}\right\rangle\right)$ and split-off $\left(\left|s o_{i}\right\rangle\right)$. Each state $|\Psi\rangle$ can be decomposed in both basis sets through projection onto the respective basis functions:

$$
\left.\left|\left\langle u_{n} \mid \psi\right\rangle\right|^{2}=|| \phi_{n}\right\rangle\left.\right|^{2} .
$$

Throughout the whole paper ||$\left.\phi_{n}\right\rangle\left.\right|^{2}$ refers to the $\left|u_{n}\right\rangle$-contribution of $|\Psi\rangle$ and the leading $\left|u_{n}\right\rangle$-contribution is referred to as the character of $|\Psi\rangle$.

For series $\mathrm{B}$ and $\mathrm{C}$ all individual QDs are modeled as truncated pyramids with a basis length of $8.76 \mathrm{~nm}$, a height $h_{\text {dot }}$ of $1.70 \mathrm{~nm}$ (6 monolayer (ML)) and an $A R$ of 0.19 . The
In-content decreases linearly (Fig. 2) in agreement with experimental investigations ${ }^{40,41}$ and theoretical findings that uniform compositions are not able to reproduce the correct electron-hole alignment ${ }^{42-44}$ and polarization properties. $^{45}$
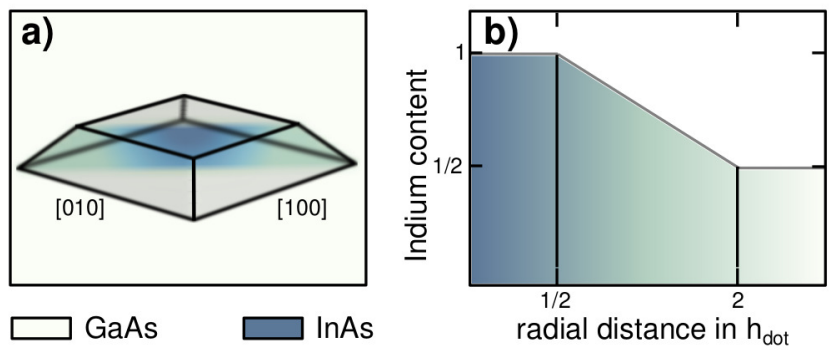

Figure 2: a) Scheme of the modeled QD geometry with a composition cross section. b) Indium content $x$ within a simulated $\mathrm{In}_{\mathrm{x}} \mathrm{Ga}_{1-\mathrm{x}}$ As QD as a function of the distance to the apex center in units of the QD's height $h_{\text {dot }}$.

Due to a lattice mismatch of about $7 \%$, the resulting heterostructure is highly strained. ${ }^{37}$ The strain shifts the energetic position of the conduction band $V_{\mathrm{CB}}$ and valence bands $V_{\mathrm{hh} / \mathrm{h}}$ via the deformation potentials $a_{\mathrm{c}}, a_{\mathrm{v}}$ and $b$. To streamline the examination of the strain-induced band coupling, we use the simplified equation of Ref. [46]:

$$
\begin{aligned}
V_{\mathrm{CB}}(r) & =E_{\mathrm{CB}}+a_{\mathrm{c}} \epsilon_{\mathrm{h}} \\
V_{\mathrm{hh} / \mathrm{lh}}(r) & =E_{\mathrm{VB}}+a_{\mathrm{v}} \epsilon_{\mathrm{h}} \pm \frac{b}{2} \epsilon_{\mathrm{b}} .
\end{aligned}
$$

Values of the deformation potentials are taken from Ref. [47].

The hydrostatic strain component $\epsilon_{\mathrm{h}}=$ $\sum_{i=x}^{z} \epsilon_{\mathrm{ii}}$ changes the energetic position of the $\mathrm{CB}$ as well as the VBs, the biaxial strain component $\epsilon_{\mathrm{b}}=\epsilon_{\mathrm{xx}}+\epsilon_{\mathrm{yy}}-2 \epsilon_{\mathrm{zz}}$ leads to a splitting of heavy-hole-band and light-hole-band. Thus, the strain along with the shape of the confinement region determines whether the ground hole state has a $|h h\rangle$ - or $|l h\rangle$-character, leading to a pure horizontal $(\mathrm{H})$ or a horizontal plus vertical $(\mathrm{V})$ polarization for the decay of the 
two particle state $\left|m_{j}^{\text {hole }}, m_{j}^{\text {electron }}\right\rangle \Longrightarrow|0\rangle$ :

$$
\begin{array}{ll}
|h h\rangle: \quad\left| \pm \frac{3}{2}, \pm \frac{1}{2}\right\rangle \stackrel{|H\rangle}{\Longrightarrow}|0\rangle \\
|l h\rangle: \quad\left| \pm \frac{1}{2}, \pm \frac{1}{2}\right\rangle \stackrel{|V\rangle+|H\rangle}{=}|0\rangle .
\end{array}
$$

Here, $m_{j}$ denotes the projection of the total angular momentum.

Analogously to the degree of polarization $(D O P)$ which has been studied in detail in the last two decades, e.g. by $\mathrm{Yu}^{1}{ }^{1} \mathrm{Kita}^{14-16}$ or Us$\operatorname{man}^{24-27,45}$ et al. we define the degree of radiation anisotropy $(D O R A)$ in three perpendicular crystallographic planes. For this purpose we evaluate the radiative recombination rate $\Gamma$ for two perpendicular propagation directions $\boldsymbol{k}$ in each plane:

$$
\begin{aligned}
& \operatorname{DORA}_{(001)}=\frac{\Gamma(\boldsymbol{k}=[110])-\Gamma(\boldsymbol{k}=[1 \overline{1} 0])}{\Gamma(\boldsymbol{k}=[110])+\Gamma(\boldsymbol{k}=[1 \overline{1} 0])} \\
& \operatorname{DORA}_{(1 \overline{1} 0)}=\frac{\Gamma(\boldsymbol{k}=[110])-\Gamma(\boldsymbol{k}=[001])}{\Gamma(\boldsymbol{k}=[110])+\Gamma(\boldsymbol{k}=[001])} \\
& \operatorname{DORA}_{(110)}=\frac{\Gamma(\boldsymbol{k}=[1 \overline{1} 0])-\Gamma(\boldsymbol{k}=[001])}{\Gamma(\boldsymbol{k}=[1 \overline{1} 0])+\Gamma(\boldsymbol{k}=[001])} .
\end{aligned}
$$

As we are using a continuum model our calculations lack the effects of interfacial symmetry and atomistic strain relaxation. Both effects influence the $p$-state splitting ${ }^{48}$ which itself may impact the spatial distribution of the groundstates. Thus, we will only concentrate on the distinction between top and edge emission and forgo a discussion of inplane properties, i.e. $D O R A_{(001)}$. A table of all $D O R A$ values can be found in the supporting informations.

From Fermi's golden rule the rate of spontaneous transitions $\Gamma_{\text {if }}$ between an initial state $|i\rangle$ and a final state $|f\rangle$ is proportional to the transition dipole moment:

$$
\Gamma_{i f}(\boldsymbol{\epsilon}) \propto|\boldsymbol{\epsilon} \cdot\langle f|\nabla| i\rangle|^{2} .
$$

In order to derive the rate of emitted photons $\Gamma_{\text {if }}$ not only with respect to their polarization $\boldsymbol{\epsilon}\left(\phi_{\epsilon}, \theta_{\epsilon}\right)$ but also as function of their prop- agation direction $\boldsymbol{k}\left(\phi_{k}, \theta_{k}\right)$, a transversal propagation is assumed. Using a frame of reference $(\varphi, \vartheta)$ with a $z$-axis aligned to the propagation direction, the integrated transition rate for all possible polarizations can be written as line integral in the $\vartheta=\pi / 2$-plane:

$$
\Gamma_{i f}(\boldsymbol{k})=\sum_{\nu}\left[\int_{0}^{2 \pi} d \varphi \Gamma_{i f}(\varphi, \pi / 2)\right] .
$$

Here, $\nu$ denotes the sum over all degenerate groundstates. The actual calculation is done in the QD's frame of reference and thus, the line integral has to be parametrized in the QD coordinates. Using the condition $\boldsymbol{\epsilon} \cdot \boldsymbol{k}=0$ the spatially resolved emission intensity is calculated as:

$$
\Gamma_{i f}(\boldsymbol{k})=\sum_{\nu}\left[\int_{\boldsymbol{\epsilon} \perp \boldsymbol{k}} d \Omega_{\epsilon}^{q d} \Gamma_{i f}(\boldsymbol{\epsilon})\right] .
$$

Here, $\phi_{k}$ and $\theta_{k}$ define the propagation direction in spherical coordinates of the QD's frame of reference.

No unique correlation between polarization and propagation direction exists. Every transition will be counted several times, if $\Gamma_{i f}(\boldsymbol{k})$ is calculated. Therefore, the rate $\Gamma_{i f}(\boldsymbol{k})$ is normalized with respect to the total rate of emitted photons.

\section{Results and Discussion}

As shown in Ref.[25], a change of the $|h h\rangle /|l h\rangle$-character of the groundstate hole wavefunction in QD stacks of different heights and different separation barrier widths causes a changing polarization. As different polarizations exhibit different propagation directions, the emission directionality changes also.

In order to understand the connection between vertical aspect ratio, the resulting biaxial strain and the emission directionality, we investigate first the emission directionality of the cuboidal QD including and excluding strain effects. The biaxial strain varies from compressive to tensile through a change of the vertical $A R$ from 0.4 to 1.6 .

To point out the connection between the ar- 
tificial cuboidal QD and more realistic geometries, two series of stacked QDs are calculated additionally, each spanning the range of compressive to tensile biaxial strain. In series B, for a constant number of five stacked QDs the role of the interdot separation is analyzed, mapping the transition from independent to closely stacked QDs with barrier widths between 10 and 0 ML. Furthermore, for a constant barrier width of $0 \mathrm{ML}$ the number of stacked QDs is varied between 1 and 11 in series C.

\section{Series A - Role of Aspect Ratio and Biaxial Strain}
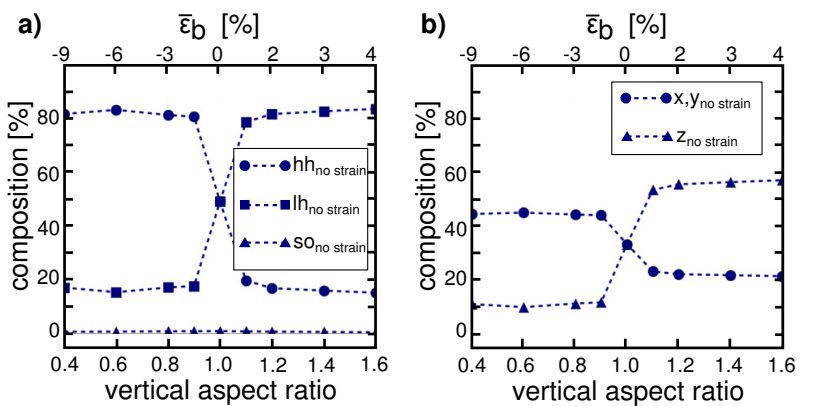

Figure 3: a) Decomposition of the hole groundstate of cuboidal InAs-QDs into $|h h\rangle$ (circle), $|l h\rangle$ (square) and $|s o\rangle$ (triangle) basis functions as well as b) decomposition into the atomic-like basis functions $|x\rangle,|y\rangle$ (circle) and $|z\rangle$ (triangle), both excluding effects of strain. For $A R=1.0$ the contributions are averaged over the four degenerate groundstates. The upper $x$-axis shows the biaxial strain expectation value for the hole groundstate.

In order to distinguish between the influence of the aspect ratio of the confinement region and the biaxial strain on the emission directionality, the series of cuboidal QDs is calculated with and without strain effects. Excluding strain means that neither direct strain related terms nor piezoelectric fields enter the $\boldsymbol{k} \cdot \boldsymbol{p}$-Hamiltonian. Although in this case the heavy-hole-band and light-hole-band stay degenerate for all $A R s,{ }^{38,39}$ already the change of the geometric properties of the confinement region changes the composition of the groundstate hole wavefunction H1 (Fig. 3).

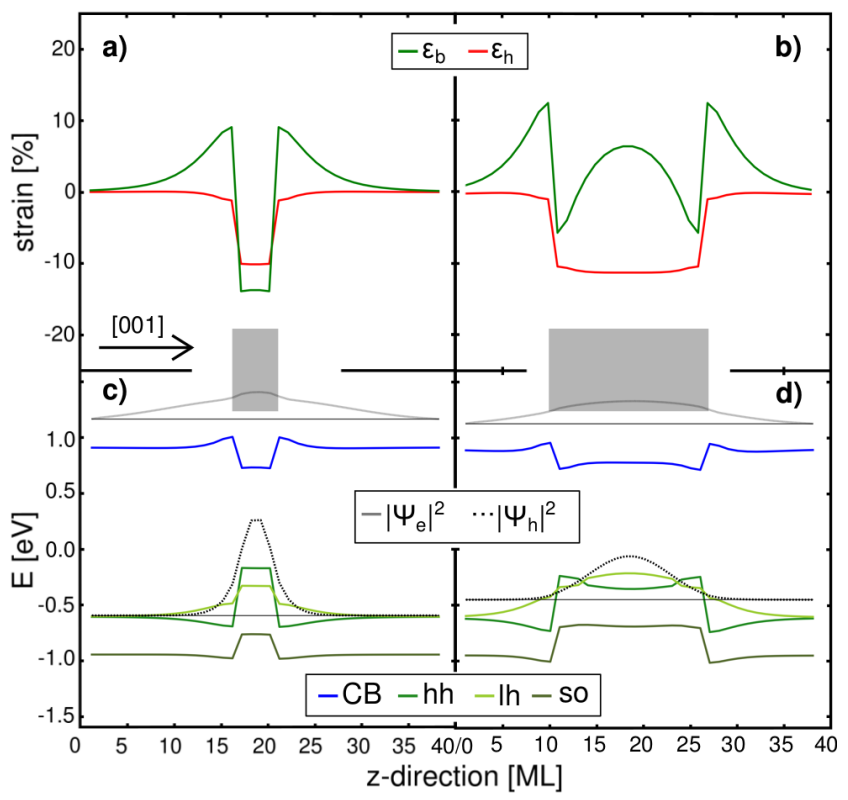

Figure 4: Distribution of biaxial strain $\left(\epsilon_{\mathrm{b}}\right)$ and hydrostatic strain $\left(\epsilon_{\mathrm{h}}\right)$ for a flat cuboidal $\mathrm{QD}$ with $A R=0.4$ (a) and a tall cuboidal QD with $A R=1.6(\mathbf{b})$. Groundstate wavefunctions of electron and hole $\left(|\psi|^{2}\right)$ and bandstructure of the spin-degenerate CBs (CB) and VB (hh, lh, so -split -off) along the [001] axis for the same ARs of 0.4 (c) and $1.6(\mathbf{d})$.

Neglecting the influence of strain the decomposition of $\mathrm{H} 1$ in the Hamiltonians eigenfunctions (Fig. 3 a) shows relatively constant $|h h\rangle$ - and $|l h\rangle$-contributions in the ranges $A R<1.0$ and $A R>1.0$ with pronounced $|h h\rangle$-and $|l h\rangle$-character respectively. The crossing of the $\mathrm{H} 1$ contributions with an equal distributed case for $A R=1.0$ can be traced back to different contributions in the atomic-like basis which are shown in Fig. 3 b. According to the transformation into the eigenstates of the Hamiltonian (Eq.3) the variation in the atomic-like basis entails a change in the $|h h\rangle$ - and $|l h\rangle$-contributions. The decomposition of $\mathrm{H} 1$ in $|x\rangle,|y\rangle$ and $|z\rangle$ reflects the prevalent confinement leading to small contributions of the $|z\rangle$ basis functions for flat QDs. The $|z\rangle$-contribution increases as the confinement in $z$-direction is reduced. For an isotropic confinement $(A R=1.0)$ the contributions of the $|x\rangle,|y\rangle$ and $|z\rangle$ basis functions are $1 / 3$ each. If the QD's extension in $z$-direction exceeds the dimension in the $x, y$-plane, the $z$-axis is the 
direction of lowest confinement and thus H1 is mainly $|z\rangle$-like.
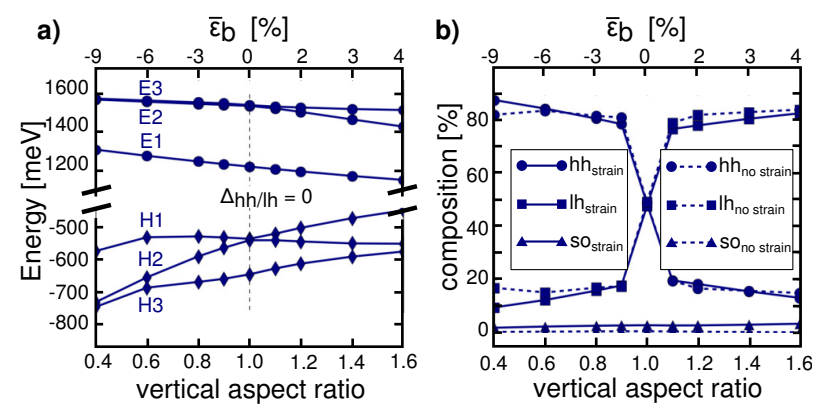

Figure 5: a) Plot of the first three spin degenerate electron (circles) and hole (diamonds) states of a cuboidal QD as function of the vertical aspect ratio. The upper $x$-axis shows the biaxial strain expectation value for the hole groundstate. The dashed vertical line at $\epsilon_{b}=0$ marks the crossing of valence-bands $|h h\rangle \leftrightarrow|l h\rangle$. b) Decomposition of the hole groundstate of cuboidal InAs-QDs into $|h h\rangle$ (circle), $|l h\rangle$ (square) and $|s o\rangle$ (triangle) basis functions including (solid line) and excluding (dashed line) strain. In the absence of the biaxial strain the contributions are averaged over the four degenerate groundstates.

In a second step, to analyze the impact of a non-vanishing strain within the QD, the strain distribution and their influence on the electronic properties is taken into account (Fig. 4). For vertical $A R s$ below 1.0 the z-component of the strain nearly vanishes within the QD. The relaxation along the growth direction is localized outside the QD in the matrix material, due to the small height of the QD. The absence of strain in $z$-direction with simultaneously compressive inplane components causes a huge negative expectation value of the biaxial strain $\bar{\epsilon}_{b}=\left\langle\Psi_{\mathrm{H} 1}\left|\epsilon_{b}\right| \Psi_{\mathrm{H} 1}\right\rangle$ (Fig. $4 \mathrm{a}$ ). Thus, the band-strain coupling (Eq.6) introduces a valence-band splitting within the QD with a raised heavy-hole-band and a lowered light-hole-band (Fig. $4 \mathrm{c}$ ).

If the vertical $A R$ is increased beyond 1.0 the $z$-component of the strain becomes larger than the inplane strain components and therefore, the biaxial strain changes sign (Fig. $4 \mathrm{~b}$ ). This also introduces a crossing in the valence-bands, meaning that the light-hole-band is raised while
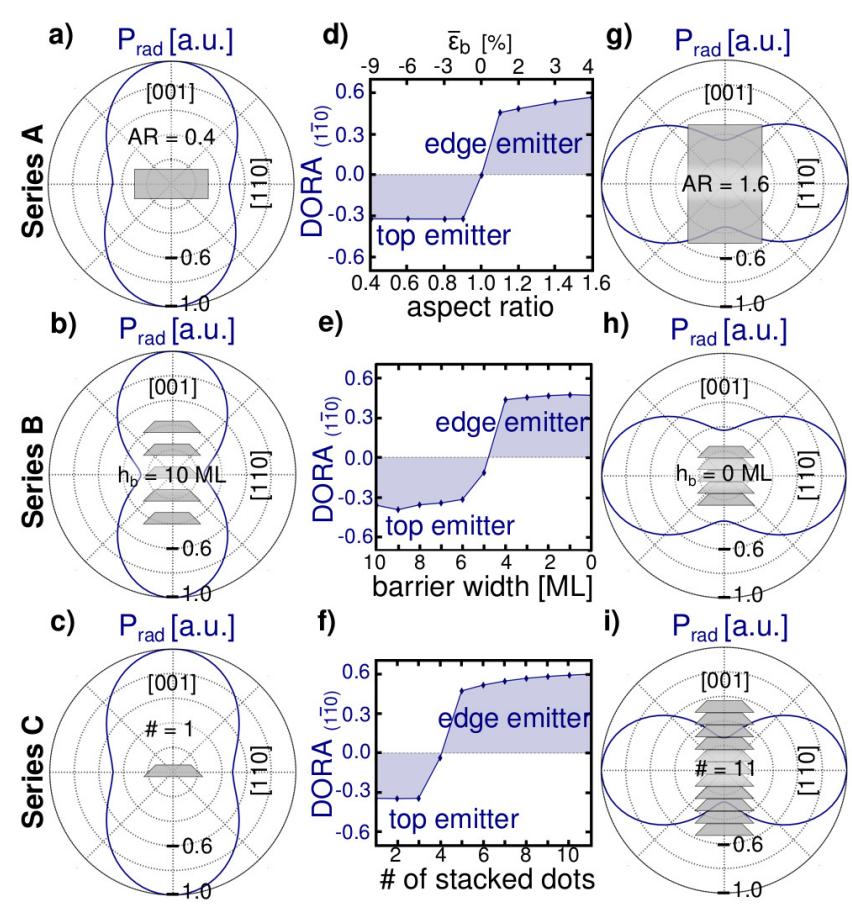

f)

i) $P_{\text {rad }}$ [a.u.]

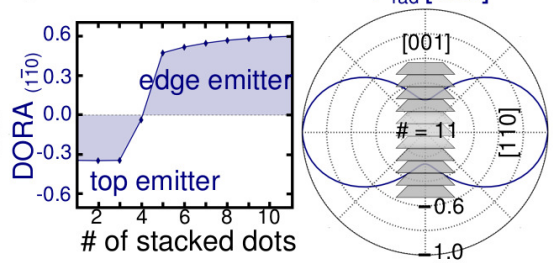

Figure 6: a)-c), g)-i) Normalized radiated power per solid angle of first and last structures within series A, B and C. d)-f) $D O R A_{(110)}$ (blue diamonds) of all structures of all series.

the heavy-hole-band is lowered (Fig. 4 d).

The evolution of the hole state energies mirrors this $A R$-dependent valence-band splitting (Fig. $5 \mathrm{a}$ ). The large biaxial strain in flat and tall QDs introduces a pronounced energetic difference between $\mathrm{H} 1$ and $\mathrm{H} 2$ which decreases as $A R=1.0$ is approached. For such an isotropic confinement the $\mathrm{H} 1 / \mathrm{H} 2$-splitting reaches zero introducing a fourfold degeneracy of the hole groundstate.

In Fig. $5 \mathrm{~b}$ the combined influence of strain and $A R$ on the composition of $\mathrm{H} 1$ is shown (solid lines) and compared to the pure $A R$-influence (dashed lines). Huo et al. ${ }^{49}$ demonstrate that the $\mathrm{H} 1$ composition of an initially strain-free QD with $|h h\rangle$-character changes only slightly if compressive biaxial strain is applied. The same is true for flat cuboidal QDs with $A R=0.4$ for which the strain increases the $|h h\rangle$-character by less than $10 \%$. For QDs in the range of $0.6 \leq A R \leq 1.0$ the $|h h\rangle$-character remains unchanged or even decreases despite reduced $|l h\rangle$-contributions. This can be explained by an increase of the $|s o\rangle$-contribution which even overcompensates the strain induced reduc- 


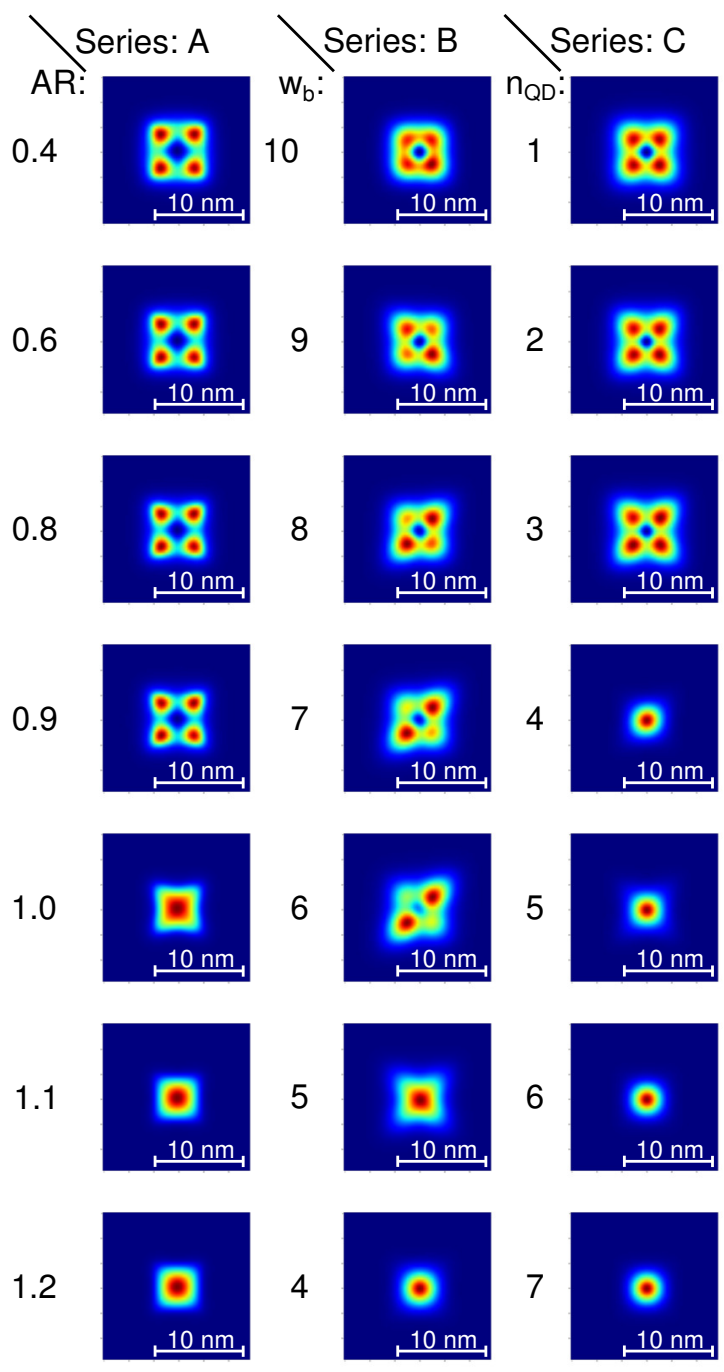

Figure 7: Sum of absolute values of the hole groundstate envelopes $|l h 1\rangle$ and $|l h 2\rangle$ in selected geometries of series $\mathrm{A}, \mathrm{B}$ and $\mathrm{C}$ in the (001)-plane integrated along the [001]-direction and normalized to the maximum in each subfigure. Structures not shown (A: $A R=1.4-1.6$; $\left.\mathrm{B}: w_{\mathrm{b}}=1-3 \mathrm{ML} ; \mathrm{C}: n_{\mathrm{QD}}=8-10\right)$ are available in the SI and resemble elements of the last row. tion of the $|l h\rangle$-contributions for $A R=0.9 . \quad$ In the isotropic case $(A R=1.0)$, the biaxial strain vanishes and thus calculations including and excluding strain show identical results with equal $|h h\rangle$-and $|l h\rangle$-contributions. One of the main results of Huo et al. ${ }^{49}$ is the change of the H1 character from $|h h\rangle$ to $|l h\rangle$ due to the application of tensile biaxial strain. Transferred to our series of $A R s$ an enhancement of the $|l h\rangle$-character of all QDs with $A R>1.0$ would be expected. However, the opposite is observed. The $A R$-induced $|l h\rangle$-character is reduced due to the inclusion of strain. This discrepancy can be explained by a different $A R$ which is around 0.6 in the mentioned work.

The impact of the change of the H1 composition onto the emission directionality is depicted in Fig. 6 a,d and g. The flat cuboidal QD shows a highly anisotropic radiation pattern (Fig. 6 a) with a DORA of $-33 \%$ (Fig. $6 \mathrm{~d}$ ). Strikingly, the emission directionality data does not reflect the increasing $|l h\rangle$-contribution in $\mathrm{H} 1$ for $A R s$ between 0.4 and 0.9. Despite the changing $|l h\rangle$-character, the DORA stays constant. This feature can be understood in terms of the symmetry change of the $|\operatorname{lh} 1\rangle$ and $|\operatorname{lh} 2\rangle$ envelopes. In Fig. 7 (first column) the sum of the absolute values of $|l h 1\rangle$ and $|l h 2\rangle$ is presented for all $A R s$ in the (001)-plane. For flat QDs the envelopes exhibit nearly perfect fourfold symmetry with a pronounced central node. As the conduction-band state E1 is always $|s\rangle$-like (Eq. 3), the overlap between the $|l h\rangle$-contribution of $\mathrm{H} 1$ and E1 nearly vanishes. ${ }^{50}$ Due to the dark $|c b\rangle \rightarrow|l h\rangle$ transition the degree of top emission is not reduced although the $|l h\rangle$-character of $\mathrm{H} 1$ increases.

In the isotropic case with $A R=1.0$ and a balanced $|h h\rangle /|l h\rangle$-character, also the QD emission directionality is isotropic, meaning DORA is zero (Fig. $6 \mathrm{~d}$ ). The $|l h\rangle$ symmetry changes to $|s\rangle$-like, brightening the $|c b\rangle \rightarrow|l h\rangle$ transition. ARs above 1.0 lead to a stepwise appearance of edge emission (Fig. $6 \mathrm{~g}$ ) and an increase of the DORA to $+46 \%$ which converges towards $+60 \%$ for the tallest QDs (Fig. 6d). The abrupt change is not only caused by the pronounced $|l h\rangle$-character but also by a symmetry change of the $|h h\rangle$ envelope which 
evolves inversely to the $|l h\rangle$-contribution (see SI), strongly reducing the overlap between the envelopes of $|h h\rangle$ and $|c b\rangle$.

\section{Series B - Role of Interdot Separa- tion}

Next, we analyze how the interdot separation $w_{\mathrm{b}}$ determines the radiation pattern of five stacked InGaAs-QDs utilizing the findings of the artificial cuboidal structures.

If QDs are separated by a $10 \mathrm{ML}$ barrier (Fig. $8 \mathrm{a}$ ), the strain distribution and bandstructure for each QD is nearly identical to a single QD (Fig. 8c), suggesting negligible interdot coupling. Inside the QDs, the hydrostatic and biaxial strain components reach maximum values of $-9 \%$ and $-13 \%$, respectively, indicating that nearly no compression along the [001]-axis is present. Thus, the systems corresponds to the already discussed flat cuboidal QD also exhibiting a heavy-hole-band raised above the light-hole-band inside the QDs. With decreasing barrier width, the central QDs are increasingly compressed along the [001]-axis (Fig. $8 \mathrm{~b}$ for $0 \mathrm{ML}$ separation) like the inner part of cuboidal QDs with ARs above 1.2.

In accordance with series $\mathrm{A}$, the emission directionality changes within the interdot separation series from predominant top emission to edge emission (Fig. 6 b,e,h). The stepwise transition as a function of the separating barrier width, i.e. interdot coupling strength, is shown in Fig. 6 e. In contrast to series $\mathrm{A}$ the emission directionality shows small variations in the DORA within the top emission regime $\left(w_{\mathrm{b}}\right.$ above $\left.5 \mathrm{ML}\right)$. Fig. 7 reveals that the $|l h\rangle$ envelopes exhibit a substantially deviating symmetry in comparison to series A. Apart from the most separated ensemble with a barrier width of $10 \mathrm{ML}$ the $|l h\rangle$ envelopes has a pronounced twofold symmetry, most probably caused by the influence of the strain induced piezoelectric field of the adjacent QDs. The lowered symmetry leads to non-vanishing $|c b\rangle \rightarrow|l h\rangle$ transitions ${ }^{50}$ and thus the emission directionality mirrors the variation in the $|l h\rangle$-contribution.

The relatively abrupt change is observed when the barrier width $w_{\mathrm{b}}$ approaches the height of the QD $h_{\mathrm{dot}}$. Thus, two regimes with pronounced anisotropic radiation properties can be distinguished which both exhibit only a minor dependence on the barrier width. The regime of weak interdot coupling $\left(h_{\mathrm{b}}>h_{\mathrm{dot}}\right)$ is associated with top emission while strong interdot coupling $\left(h_{\mathrm{b}}<h_{\mathrm{dot}}\right)$ leads to edge emission. If the barrier width is of the order of the QDs' height the stack neither exhibits a dominating top emission nor dominating edge emission but is in the very narrow transition regime, where $D O R A$ reach minimum values of $+11 \%$ for $w_{\mathrm{b}}=5 \mathrm{ML}$. A table of all DORA values can be found in supporting informations.

\section{Series C - Role of Number of Stacked QDs}

In the last series we focus on the number of stacked QDs. We choose to set $w_{\mathrm{b}}$ to zero as experimental findings suggest that columnar QDs which were grown with a nominal barrier width of a few ML stay in contact to each other along the growth direction. ${ }^{14-16}$ Furthermore, the choice of $w_{\mathrm{b}}=0$ is of particular interest as it represents the maximal interdot coupling. Close stacking of QDs corresponds directly to increasing the vertical $A R$ of the cuboidal QDs in series A.

Reflecting the increasing influence of the light-hole-band on the hole groundstate, stacking transforms the top emitting single QD (Fig. 6c) into an edge emitting stack (Fig. 6i). Although the interdot coupling stays the same for all QDs within the stacking series, we again observe a sudden change in emission anisotropy resembling the change of symmetry of the $|l h\rangle$ envelopes as seen in series A. Ensembles with less than four QDs show a pronounced DORA with values around $-34 \%$ while stacks with more than four QDs exhibit values above $+50 \%$ which again converge to $+60 \%$ for higher number of stacked dots (Fig. $6 \mathrm{f}$ ).

Defining the collective aspect ratio $A R_{\text {col }}\left(n_{\mathrm{QD}}\right)$ 

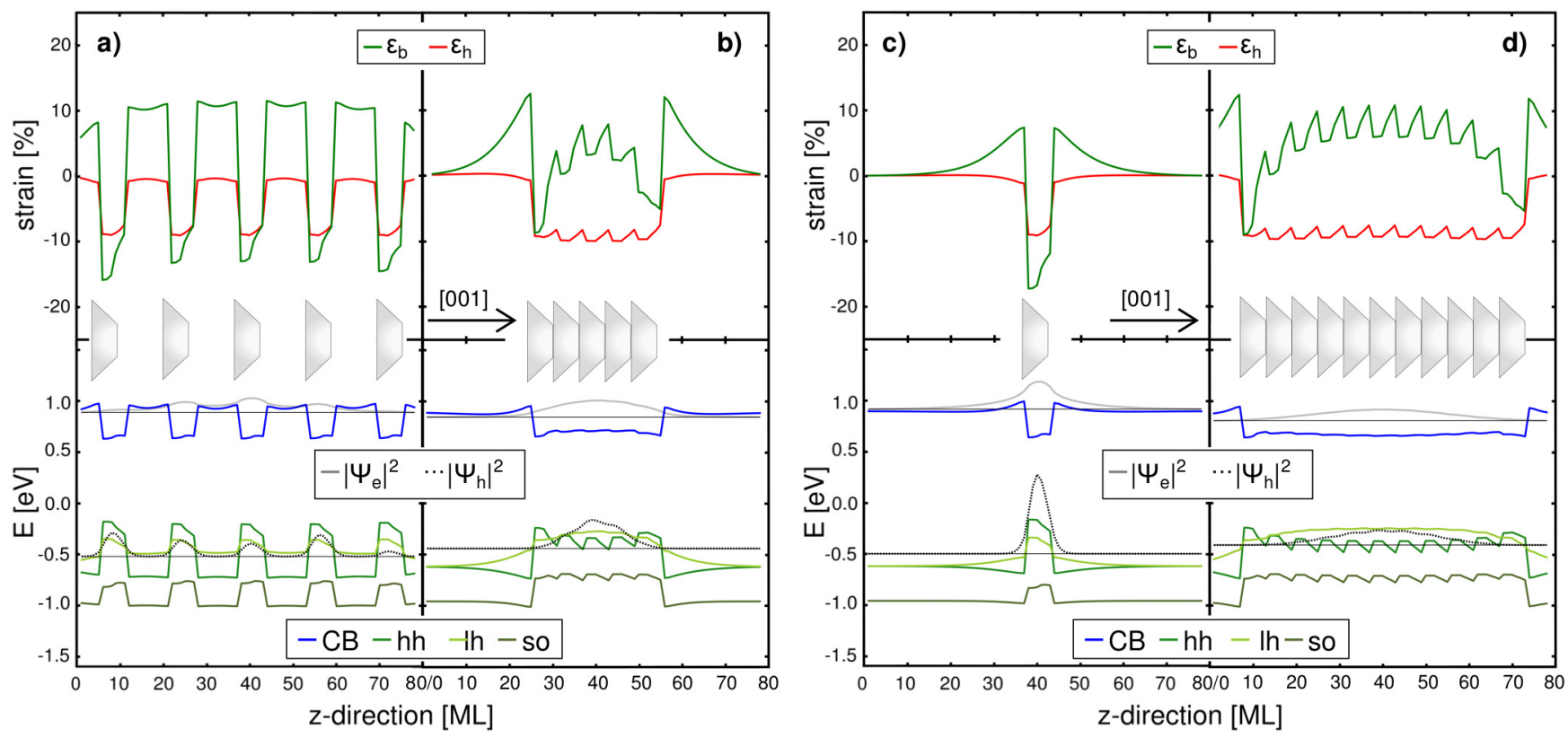

Figure 8: Distribution of biaxial strain $\left(\epsilon_{\mathrm{b}}\right)$ and hydrostatic strain $\left(\epsilon_{\mathrm{h}}\right)$ for a five stacked InGaAs-QDs with $w_{\mathrm{b}}=10 \mathrm{ML}(\mathbf{a})$ and $w_{\mathrm{b}}=0 \mathrm{ML}(\mathbf{b})$ as well as for a single InGaAs-QD (c) and eleven stacked InGaAs-QDs with $w_{\mathrm{b}}=0 \mathrm{ML}(\mathbf{d})$. Groundstate wavefunctions of electron and hole $\left(|\psi|^{2}\right)$ and bandstructure of the spin-degenerate CBs (CB) and VB (hh, lh, so -split-off) along the [001] axis for the same structures.

of $n_{\mathrm{QD}}$ stacked QDs

$$
A R_{\mathrm{col}}\left(n_{\mathrm{QD}}\right)=n_{\mathrm{QD}} \cdot h_{\mathrm{dot}} /(\text { average width })
$$

allows us to connect our findings to the cuboidal QDs of series A. The parameter $A R_{\text {col }}\left(n_{\mathrm{QD}}\right)$ determines the radiation anisotropy in the same way as the vertical $A R$ does in the cuboidal case. Thus, nearly isotropic radiation (DORA $=-0.04)$ characteristics are found for the stack of four QDs, that most closely realizes an isotropic confinement $\left(A R_{\text {col }}(4)=0.92\right)$.

\section{Conclusion}

We show that the emission directionality of InGaAs-QDs can be engineered via the vertical aspect ratio of the confinement region. Using a series of artificial cuboidal QDs we analyze the relation between aspect ratio and strain distribution as well as the question how combined influence of them determines the $|h h\rangle /|l h\rangle$-character of the groundstate hole wavefunction and thus the emission directionality.
Two series of stacked InGaAs-QDs are investigated, firstly with a variation of the barrier width, secondly with a variation of the number of stacked dots in order to proof that the findings of the artifical cuboidals QDs are also valid for realistic structures. The calculations reveal qualitative changes of the radiation pattern and provide quantitative values of the degree of radiation anisotropy (DORA) for all considered parameters.

We confirm the findings of Huo et al. ${ }^{50}$ that the change of optical properties is connected to a change of the $|l h\rangle$-symmetry which brightens the $|c b\rangle \rightarrow|l h\rangle$ transition.

Our results give important insights regarding the design of photonic devices based on coupled QDs. The data help to tune the lateral and vertical interaction of stacked QDs as they present how the single stacks can be designed either as edge or top emitter.

\section{Author Information}

\section{Corresponding Author}

* lath@physik.tu-berlin.de 


\section{ORCID}

Ludwig A. Th. Greif: 0000-0002-6732-7062

Stefan T. Jagsch:

Markus R. Wagner: 0000-0002-7367-5629

Andrei Schliwa: $\quad$ 0000-0001-7085-3680

Notes

The authors declare no competing financial interest.

\section{Acknowledgement}

The authors thank Christoph Tyborski for the valuable discussions about the manuscript and the Deutsche Forschungsgemeinschaft (DFG) which founded this work in the frame of SFB 787 .

Supporting Information Available: Sum of light-hole envelopes and heavy-hole envelopes of the groundstate hole wavefunction in all geometries of series A, B and C in the (001)-plane and (100)-plane. Tables of calculated DORA data in the (001)-,(110)-and (110)-plane of series $\mathrm{A}, \mathrm{B}$ and $\mathrm{C}$. This material is available free of charge via the Internet at http: //pubs . acs . org/.

\section{References}

(1) Yu, P. et al. Optical Anisotropy in Vertically Coupled Quantum Dots. Phys. Rev. B 1999, 60, 680-685.

(2) Kumar, J.; Kapoor, S.; Gupta, S. K.; Sen, P. K. Theoretical Investigation of the Effect of Asymmetry on Optical Anisotropy and Electronic Structure of Stranski-Krastanov Quantum Dots. Phys. Rev. B 2006, 74, 115326.

(3) Favero, I.; Cassabois, G.; Jankovic, A.; Ferreira, R.; Darson, D.; Voisin, C.; Delalande, C.; Ph, R.; Badolato, A.; Petroff, P. M.; Gerard, J. M. Giant Optical Anisotropy in a Single InAs Quantum Dot in a very Dilute Quantum-Dot ensemble. Applied Physics Letters 2005, 86, 41904.

(4) Asada, M.; Miyamoto, Y.; Suematsu, Y. Gain and the Threshold of Three-
Dimensional Quantum-Box Lasers. IEEE J. of Quantum Electron. 1986, 22, 19151921.

(5) Arakawa, Y.; Sakaki, H. Multidimensional Quantum Well Laser and Temperature Dependence of its Threshold Current. Appl. Phys. Lett. 1982, 40, 939-941.

(6) Arakawa, Y.; Yariv, A. Quantum Well Lasers - Gain, Spectral, Dynamics. IEEE J. of Quantum Electron. 1986, 22, 18871899 .

(7) Mecozzi, A.; Piazzolla, S.; D’Ottavi, A.; Spano, P. Passage Time Statistics in Semiconductor Laser Turn On. Phys. Rev. A 1988, 38, 3136-3138.

(8) Lounis, B.; Orrit, M. Single-Photon Sources. Rep. Prog. Phys. 2005, 68, 1129 1179 .

(9) Ding, X.; He, Y.; Duan, Z. C.; Gregersen, N.; Chen, M. C.; Unsleber, S.; Maier, S.; Schneider, C.; Kamp, M.; Höfling, S.; Lu, C. Y.; Pan, J. W. OnDemand Single Photons with High Extraction Efficiency and Near-Unity Indistinguishability from a Resonantly Driven Quantum Dot in a Micropillar. Phys. Rev. Lett. 2016, 116, 20401.

(10) Stier, O.; Grundmann, M.; Bimberg, D. Electronic and Optical Properties of Strained Quantum Dots Modeled by 8Band kp Theory. Phys. Rev. B 1999, 59, 5688-5701.

(11) Akiyama, T.; Sugawara, M.; Arakawa, Y. Quantum-Dot Semiconductor Optical Amplifiers. Proc. IEEE 2007, 95, 1757-1766.

(12) Sakaki, H. Scattering Suppression and High-Mobility Effect of Size-Quantized Electrons in Ultrafine Semiconductor Wire Structures. Jpn. J. Appl. Phys. 1980, 19, 735-738.

(13) Sakaki, H. High Mobility Effect of Electrons in Ultrafine Semiconductor Wire 
Structures. J. Vac. Sci. Technol. 1981, 19, 148-149.

(14) Kita, T.; Wada, O.; Ebe, H.; Nakata, Y.; Sugawara, M. Polarization-Independent Photoluminescence from Columnar InAs/GaAs Self-Assembled Quantum Dots. Jpn. J. Appl. Phys. 2002, 41, 1143-1145.

(15) Kita, T.; Jayavel, P.; Wada, O.; Ebe, H.; Nakata, Y.; Sugawara, M. Polarization Controlled Edge Emission from Columnar InAs/GaAs Self-Assembled Quantum Dots. Physica Status Solidi (C) 2003, 1140, 1137-1140.

(16) Kita, T.; Tamura, N.; Wada, O.; Sugawara, M.; Nakata, Y.; Ebe, H.; Arakawa, Y. Artificial Control of Optical Gain Polarization by Stacking Quantum Dot Layers. Appl. Phys. Lett. 2006, 88, 211106.

(17) Ridha, P.; Li, L.; Rossetti, M.; Patriarche, G.; Fiore, A. Polarization Dependence of Electroluminescence from Closely-Stacked and Columnar Quantum Dots. Opt. Quant. Electron. 2008, 40, 239-248.

(18) Ikeuchi, Y.; Inoue, T.; Asada, M.; Harada, Y.; Kita, T.; Taguchi, E.; Yasuda, H. Multidirectional Observation of Photoluminescence Polarization Anisotropy in Closely Stacked InAs/GaAs Quantum Dots. Appl. Phys. Expr. 2011, 4, 62001 .

(19) Yasuoka, N.; Ebe, H.; Kawaguchi, K.; Ekawa, M.; Sekiguchi, S.; Morito, K.; Wada, O.; Sugawara, M.; Arakawa, Y. Polarization-Insensitive Quantum Dot Semiconductor Optical Amplifiers using Strain-Controlled Columnar Quantum Dots. J. Lightwave Technol. 2012, 30, 68-75.

(20) Chokamnuai, T.; Rattanadon, P.; Thainoi, S.; Panyakeow, S.; Kanjanachuchai, S. Polarization Anisotropy of Stacked InAs Quantum Dots on InGaAs/GaAs Cross-Hatch Patterns. J. Cryst. Growth 2013, 378, 524-528.

(21) He, J.; Krenner, H. J.; Pryor, C.; Zhang, J. P.; Wu, Y.; Allen, D. G.; Morris, C. M.; Sherwin, M. S.; Petroff, P. M. Growth, Structural, and Optical Properties of Self-Assembled (In,Ga)As Quantum Posts on GaAs. Nano Lett. 2007, 7, 802-806.

(22) Saito, T.; Ebe, H.; Arakawa, Y.; Kakitsuka, T.; Sugawara, M. Optical Polarization in Columnar InAs/GaAs Quantum Dots: 8-Band kp Calculations. Phys. Rev. B 2008, 77, 195318.

(23) Ridha, P.; Li, L. H.; Mexis, M.; Smowton, P. M.; Andrzejewski, J.; Sek, G.; Misiewicz, J.; O'Reilly, E. P.; Patriarche, G.; Fiore, A. Polarization Properties of Columnar Quantum Dots: Effects of Aspect Ratio and Compositional Contrast. IEEE J. Quant Electron. 2010, 46, 197-204.

(24) Usman, M. In-Plane Polarization Anisotropy of Ground State Optical Intensity in InAs/GaAs Quantum Dots. J. Appl. Phys. 2011, 110, 94512.

(25) Usman, M.; Inoue, T.; Harda, Y.; Klimeck, G.; Kita, T. Experimental and Atomistic Theoretical Study of Degree of Polarization from Multilayer InAs/GaAs Quantum Dot Stacks. Phys. Rev. B 2011, 84, 115321 .

(26) Usman, M.; Tan, Y. H. M.; Ryu, H.; Ahmed, S. S.; Krenner, H. J.; Boykin, T. B.; Klimeck, G. Quantitative Excited State Spectroscopy of a Single InGaAs Quantum Dot Molecule through Multi-Million-Atom Electronic Structure Calculations. Nanotechnology 2011, 22, 315709.

(27) Usman, M. Atomistic Theoretical Study of Electronic and Polarization Properties of Single and Vertically Stacked Elliptical 
InAs Quantum Dots. Phys. Rev. B 2012, 86,155444 .

(28) Giessen, H.; Lippitz, M. Directing Light Emission from Quantum Dots. Science 2010, 329, 910-911.

(29) Akimov, A. V.; Mukherjee, A.; Yu, C. L.; Chang, D. E.; Zibrov, A. S.; Hemmer, P. R.; Park, H.; Lukin, M. D. Generation of Single Optical Plasmons in Metallic Nanowires Coupled to Quantum Dots. Nature 2007, 450, 402-406.

(30) Zadeh, I. E.; Elshaari, A. W.; Jöns, K. D.; Fognini, A.; Dalacu, D.; Poole, P. J.; Reimer, M. E.; Zwiller, V. Deterministic Integration of Single Photon Sources in Silicon Based Photonic Circuits. Nano Lett. 2016, 16, 2289-2294.

(31) Ganesh, N.; Zhang, W.; Mathias, P. C.; Chow, E.; Soares, J. a. N. T.; Malyarchuk, V.; Smith, A. D.; Cunningham, B. T. Enhanced Fluorescence Emission from Quantum Dots on a Photonic Crystal Surface. Nat. Nanotechnol. 2007, 2, 515-520.

(32) Noda, S.; Fujita, M.; Asano, T. Spontaneous-Emission Control by Photonic Crystals and Nanocavities. Nat. Photonics 2007, 1, 449-458.

(33) Xie, Q. H.; Madhukar, A.; Chen, P.; Kobayashi, N. P. Vertically Self-Organized InAs Quantum Box Islands on GaAs(100). Physical Review Letters 1995, 75, 25422545.

(34) Li, L. H.; Ridha, P.; Patriarche, G.; Chauvin, N.; Fiore, A. Shape-Engineered Epitaxial InGaAs Quantum Rods for Laser Applications. Appl. Phys. Lett. 2008, 92, 121102.

(35) Yuan, X.; Weihausen-Brinkmann, F.; Martín-Sánchez, J.; Piredda, G.; Křápek, V.; Huo, Y.; Huang, H.; Schimpf, C.; Schmidt, O. G.; Edlinger, J.; Bester, G.; Trotta, R.; Rastelli, A. StrainDriven Reorientation of the Quantization
Axis of a Quantum Dot for Integrated Quantum Photonics. Nat. Comm. 2018, 9, 3058

(36) Schliwa, A.; Winkelnkemper, M.; Bimberg, D. Impact of Size, Shape, and Composition on Piezoelectric Effects and Electronic Properties of $\operatorname{In}(\mathrm{Ga}) \mathrm{As} / \mathrm{GaAs}$ Quantum Dots. Phys. Rev. B 2007, 76, 205324.

(37) Grundmann, M.; Stier, O.; Bimberg, D. InAs/GaAs pyramidal quantum dots: Strain distribution, optical phonons, and electronic structure. Phys. Rev. B 1995, 52, 11969-11981.

(38) Enders, P.; Bärwolff, a.; Woerner, M.; Suisky, D. kp Theory of Energy Bands, Wave Functions, and Optical Selection Rules in Strained Tetrahedral Semiconductors. Physical Review B 1995, 51, 16695-16704.

(39) Chuang, S. L. Efficient Band-Structure Calculations of Strained Quantum Wells. Phys. Rev. B 1991, 43, 9649-9661.

(40) Bruls, D. M.; Vugs, J. W. A. M.; Koenraad, P. M.; Salemink, H. W. M.; Wolter, J. H.; Hopkinson, M.; Skolnick, M. S.; Long, F.; Gill, S. P. A. Determination of the Shape and Indium Distribution of Low-Growth-Rate InAs Quantum Dots by Cross-Sectional Scanning Tunneling Microscopy. Appl. Phys. Lett. 2002, 81, 1708-1710.

(41) Lemaître, A.; Patriarche, G.; Glas, F. Composition Profiling of InAs/GaAs Quantum Dots. Appl. Phys. Lett. 2004, 85, 3717-3719.

(42) Fry, P. W. et al. Inverted Electron-Hole Alignment in InAs-GaAs Self-Assembled Quantum Dots. Phys. Rev. Lett. 2000, 84, 733-736.

(43) Barker, J. A.; O'Reilly, E. P. Theoretical Analysis of Electron-Hole Alignment in InAs-GaAs Quantum Dots. Phys. Rev. B 2000, 61, 13840-13851. 
(44) Sabathil, M.; Hackenbuchner, S.; Birner, S.; Majewski, J. A.; Vogl, P.; Finley, J. J. Theory of Vertical and Lateral Stark Shifts of Excitons in Quantum Dots. Phys. Status Solidi C 2003, 1184, 1181-1184.

(45) Usman, M.; Tasco, V.; Todaro, M. T.; De Giorgi, M.; Oreilly, E. P.; Klimeck, G.; Passaseo, A. The Polarization Response in InAs Quantum Dots: Theoretical Correlation Between Composition and Electronic Properties. Nanotechnology 2012, 23, 165202.

(46) Chuang, S. L. Physics of Optoelectronic Devices; Wiley: New York, 1995.

(47) Vurgaftman, I.; Meyer, J. R.; RamMohan, L. R. Band parameters for III$\mathrm{V}$ compound semiconductors and their alloys. J. Appl. Phys. 2001, 89, 5815-5875.

(48) Bester, G.; Zunger, A. Cylindrically Shaped Zincblende Semiconductor Quantum Dots do not have Cylindrical Symmetry: Atomistic Symmetry, Atomic Relaxation, and Piezoelectric Effects. Phys. Rev. B 2005, 71, 45318.

(49) Huo, Y. H. et al. A Light-Hole Exciton in a Quantum Dot. Nat. Phys. 2013, 10, 4651 .

(50) Huo, Y. H.; Křápek, V.; Schmidt, O. G.; Rastelli, A. Spontaneous Brightening of Dark Excitons in GaAs/AlGaAs Quantum Dots near a Cleaved Facet. Phys. Rev. B 2017, 95, 165304. 


\section{For Table of Contents Use Only}

Title:

Tuning the Emission Directionality of Stacked Quantum Dots

\section{Authors:}

Ludwig A. Th. Greif; Stefan. T. Jagsch; Markus. R. Wagner; Andrei. Schliwa

Synopsis:

The radiation pattern of single and stacked QDs shows a pronounced anisotropy. In the present work, we use 8-band $\boldsymbol{k} \cdot \boldsymbol{p}$-theory to demonstrate that the interdot coupling strength (via the separating barrier width) and the number of stacked QDs are effective parameters for adjusting the emission directionality. Our work helps to tune the interaction of vertically and laterally arranged QDs which constitute a popular element of photonic devices in a wide range of fields as nano-photonics, laser design and quantum electronics.

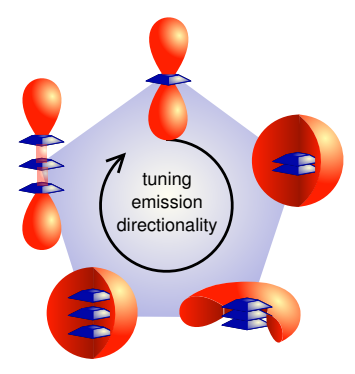

